\title{
Isolated Axillary Artery Aneurysm Causing Peripheral Neuropathy of Upper Limb - A Case Report
}

\author{
Muralikrishna Nekkanti*, Karthikeyan Sivagnanam and Seetharama Bhat
}

Associate Professor - Vascular Surgery, DNB Vascular Surgery, Sri Jayadeva Institute of Cardiovascular Science and Research, India

\begin{abstract}
Axillary artery aneurysms are very rare and frequently occur as a result of penetrating or blunt chest trauma. They also occur iatrogenically or as a post obstructive lesion due to thoracic outlet syndrome or secondary to the chronic use of crutches. Atherosclerotic axillary aneurysms are very rare. When diagnosed, they must be treated without delay as they may invariably cause distal embolism or peripheral neuropathy threatening the affected limb.

We describe a case of isolated true axillary artery aneurysm that had thrombosed and the patient presented with neuropathic symptoms. Duplex scan and CT angiogram were done to confirm the diagnosis which was successfully treated by axillary artery to proximal brachial artery interposition bypass with reversed saphenous vein graft.
\end{abstract}

Keywords: Aneurysm; Neuropathy; Brachial artery

\section{Introduction}

Axillary artery aneurysms are a rare phenomenon. Mostly, the aetiology is due to penetrating or blunt chest trauma or secondary to chronic use of crutches. They also occur iatrogenically or due to post obstructive lesion in thoracic outlet syndrome. Atherosclerotic axillary artery aneurysms are very rare. Surgical treatment without delay is very important as it causes ischemic symptoms of the hand endangering the affected limb or neuropathic complications affecting the function of the limb. We present a thrombosed axillary artery aneurysm with neuropathic complication for which a successful axillary to brachial artery interposition bypass was done with reversed saphenous vein graft.

\section{Case Report}

An 18 years old female presented with numbness and weakness of right upper limb associated with pain in axilla for one month duration. There was no history of trauma. She was on treatment for aorto-arteritis and had a previous renal angioplasty for accelerated hypertension. Presently, her serum creatinine was normal and CT angiogram of right upper limb revealed a thrombosed fusiform axillary artery aneurysm involving the third part with good reformation distally (Figures 1 and 2). She underwent excision of the aneurysm with end to end interposition bypass grafting from distal axillary artery to proximal

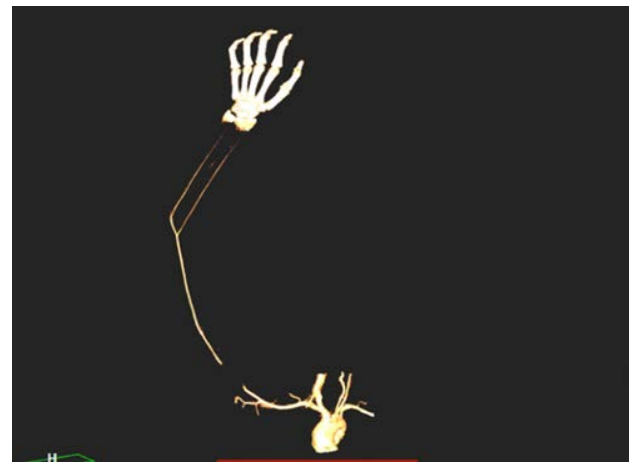

Figure 1: CT angiogram of right upper limb showing thrombosed axillary artery aneurysm with good reformation. brachial artery with reversed saphenous vein graft. We approached the aneurysm through an infra-clavicular transverse incision extending from the anterior axillary fold to medial part of the arm in the axilla. The pectoralis major and minor muscles were retracted without cutting. Branches of brachial plexus were adhered to the aneurysm sac which was dissected ensuring continuity and interposition bypass grafting was performed (Figures 3 and 4). Post-operatively her symptoms improved well and the limb movements are improving with physiotherapy.

\section{Discussion}

Peripheral aneurysms occurring in the subclavian-axillary segment account for less than $1 \%$ of all aneurysms according to Hobson et al. [1] of which $88 \%$ of them occurred in the subclavian artery (Figure 5). Aneurysms of the axillary artery are mostly caused due to repetitive trauma in crutch users [2], baseball pitchers [3]. They also occur

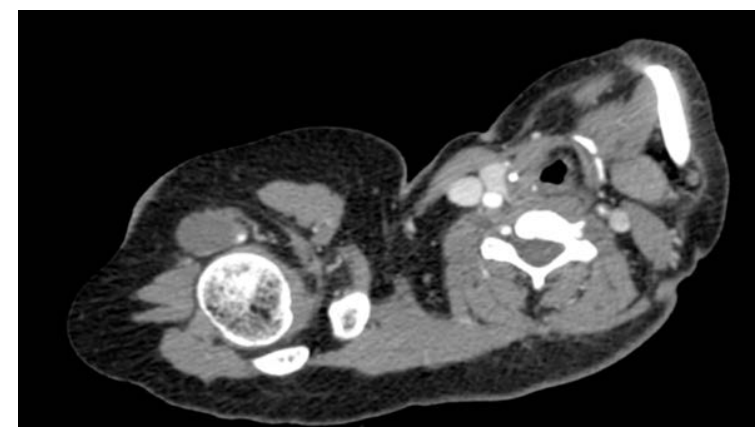

Figure 2: Thrombosed axillary aneurysm on coronal section

*Corresponding author: Dr. Karthikeyan Sivagnanam, Department of CTVS, Sri Jayadeva Institute of Cardiovascular Sciences \& Research, India, Tel: 91-8022977400; E-mail: karthiksivagnanam@outlook.com

Received May 28, 2015; Accepted June 26, 2015; Published June 28, 2015

Citation: Nekkanti M, Sivagnanam K, Bhat S (2015) Isolated Axillary Artery Aneurysm Causing Peripheral Neuropathy of Upper Limb - A Case Report. J Vasc Med Surg 3: 207. doi:10.4172/2329-6925.1000207

Copyright: @ 2015 Nekkanti M, et al. This is an open-access article distributed under the terms of the Creative Commons Attribution License, which permits unrestricted use, distribution, and reproduction in any medium, provided the original author and source are credited. 


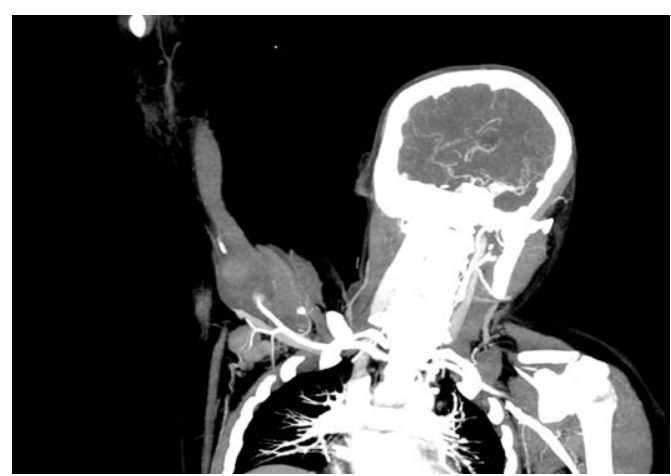

Figure 3: Sagittal section showing the axillary artery aneurysm.

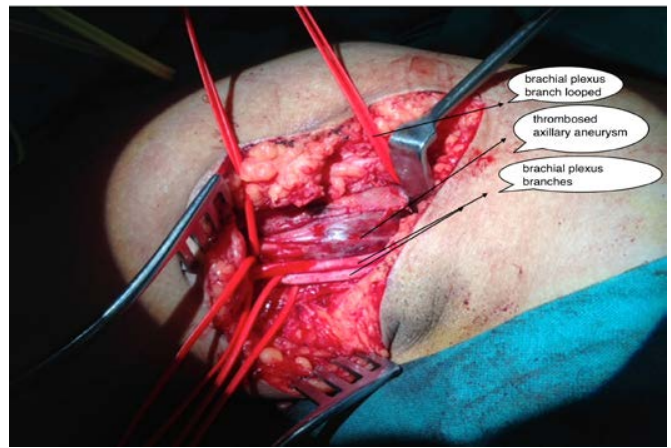

Figure 4: Operative picture showing dissected axillary artery aneurysm with cords of brachial plexus.

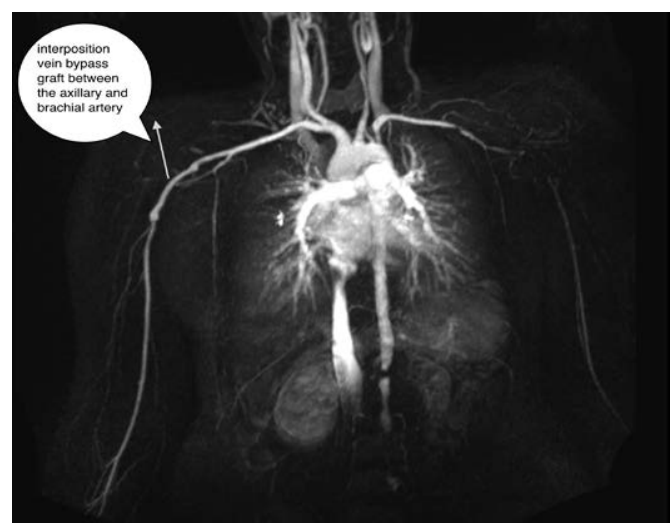

Figure 5: MRA TOF sequence showing the interposition bypass graft between axillary artery and brachial artery. iatrogenically or as a post obstructive lesion due to thoracic outlet syndrome. Atherosclerotic axillary aneurysms are very rare. They can also be due to infectious cause, congenital, or due to marfan's syndrome. These aneurysms cause neurologic defects due to compression of the brachial plexus. They can also cause thromboembolic symptoms and ischemia threatening the affected extremity.

Diagnosis of axillary artery aneurysms is difficult clinically due to the location. Due to the pressure effects on the adjacent nerves, they are difficult to palpate. Mostly they present with weakness of the involved limb or limb ischemia secondary to thromboembolism or thrombotic occlusion.

They can be managed by surgical or endovascular approach though surgical method is considered a better choice. One of the main objectives with surgical method is to prevent injury to the brachial plexus, due to the proximity of the branches and also adhesions due to the inflammation around the aneurysm.

When diagnosed these aneurysms should be treated promptly as they may cause ischemic or neuropathic complications.

\section{References}

1. Hobson II RW, Israel MR, Lynch TG (1982) Axilo-subclavian arterial aneurysms Aneurisms - Diagnosis and Treatment. Grune and Stratton, New York, USA 435 .

2. Tripp HF, Cook JW (1998) Axillary artery aneurysms. Mil Med 163: 653-655.

3. Schneider K, Kasparyan NG, Altchek DW, Fantini GA, Weiland AJ (1999) An aneurysm involving the axillary artery and its branch vessels in a major league baseball pitcher: A case report and review of the literature. Am J Sports Med 27: 370-375. 